\title{
Nonketotic hyperglycemia induced occipital lobe seizures: a case report
}

\author{
Samantha Richardson, BS ${ }^{1}$, Sona K. Shah, MD ${ }^{1}$
}

\section{ABSTRACT}

Typically, nonketotic hyperglycemia induces partial motor seizures. We present a rare case of nonketotic hyperglycemia induced occipital lobe seizures and discuss the impact of hemoglobin A1c on seizure activity, typical imaging results, and use of antiepileptic drugs in management.
Author affiliations are listed at the end of this article.

Correspondence to: Samantha Richardson, BS Marshall University Joan C. Edwards School of Medicine richardso161@marshall.edu

\section{KEYWORDS}

occipital lobe, epilepsy, seizure, nonketotic hyperglycemia

\section{INTRODUCTION}

Occipital lobe epilepsy accounts for five to ten percent of all epilepsies. 1 While idiopathic occipital lobe epilepsy has an age of onset in childhood or adolescence,2 symptomatic occipital lobe seizures may start at any age depending on the underlying disorder provoking seizure onset. ${ }^{1}$ Clinical manifestations include elementary and complex visual hallucinations, ictal amaurosis, visual illusions, sensory hallucinations of ocular movements, oculomotor symptoms, nystagmus, and repetitive eye closure or eyelid fluttering. ${ }^{1}$ Occipital seizures may be provoked by multiple etiologies including posterior reversible encephalopathy syndrome (PRES), metabolic encephalopathies, malformations of cortical development, trauma, vascular disturbances, tumors, or infection/ inflammation. ${ }^{2} \mathrm{~A}$ rare cause of provoked occipital seizures is nonketotic hyperglycemia, a syndrome consisting of severe hyperglycemia, hyperosmolality, and intracellular dehydration without ketoacidosis. ${ }^{3}$ Nonketotic hyperglycemia often causes partial motor seizures with or without secondary generalization; however, there have been a few reported cases of nonketotic hyperglycemia provoking occipital lobe seizures. ${ }^{4}$

\section{CASE REPORT}

A previously healthy 59-year-old man with no past medical history and no primary care physician presented to the emergency department (ED) with acute onset headache for the past four days with associated left lower field visual changes and metamorphopsia. Vitals were within normal limits except for increased blood pressures as high as $218 / 105$. CT scan with and without contrast at this time showed no acute intracranial process with a probable old lacunar infarct of the left thalamus. His labs were within normal limits except for an elevated glucose of 242 and white blood cell count of 14.8. His hemoglobin $\mathrm{A} 1 \mathrm{c}(\mathrm{HbA} 1 \mathrm{C})$ was not evaluated, and he left against medical advice.

The patient returned to the ED two days later with hypertension and left hemianopsia. His blood pressure was 195/98. Repeat CT angiography showed 
$6.3 \mathrm{~mm}$ left sided cavernous carotid aneurysm and increased mean transit time (MTT). The next day, the patient complained of blurry vision and left eye deviation with visual hallucinations consisting of two green and red pinwheels floating from the left to the right of his visual field. A stat electroencephalography (EEG) was ordered which showed six seizures originating from the right occipital lobe. Patient also hallucinated various types of dogs, children, and men during seizures. Patient had 13 partial right occipital lobe seizures in 74 minutes. He was given $4 \mathrm{mg}$ Lorazepam, $1000 \mathrm{mg}$ Valproate, and 1000 mg Levetiracetam twice daily with no change in seizure frequency. He continued to have 11 seizures over the next 90 minutes, each around 40 seconds in length. He was given another $4 \mathrm{mg}$ Lorazepam. Levetiracetam was increased to $1300 \mathrm{mg}$ every eight hours and Valproate was increased to $500 \mathrm{mg}$ every six hours when he continued to seize the following day. He was intubated and sedated with Midazolam at this time. Seizure activity slowed to two seizures per hour then gradually to one per hour. The patient was seizure free by the third day of hospitalization. His glucose level decreased to the high 100's for the rest of his hospital stay. Patient was discharged on day 12. On follow up, he continued $1000 \mathrm{mg}$ Valproate and $2000 \mathrm{mg}$ Levetiracetam twice daily. He has been seizure free for fifteen months. His type 2 diabetes (T2DM) was treated and he has maintained a $\mathrm{HbA1c}$ under $7.8 \%$ since discharge.

\section{DISCUSSION}

Onset of epileptic seizures over age 50 is highly suggestive of a lesion or metabolically provoked process. ${ }^{5}$ Seizures related to nonketotic hyperglycemia are typically partial seizures that are refractory to antiepileptic drugs (AED) and resolve with treatment of hyperglycemia. ${ }^{6}$ Although our patient had only mildly elevated glucose levels on admission, his $\mathrm{HbA} 1 \mathrm{c}$ was highly elevated at $14.8 \%$. This is in agreement with case reports which conclude that long standing hyperglycemia, rather than an acute rise in glucose levels, is the likely cause of seizure onset. ${ }^{4,7}$ In fact, half of patients presenting with epileptic seizures in the setting of nonketotic hyperglycemia have undiagnosed diabetes, ${ }^{5}$ as seen in our patient.
On admission, our patient was found to have prolonged MTT in posterior cerebral arteries bilaterally. The ictal state is most associated with increased perfusion to focal area of epileptiform activity, while the postictal state is associated with focal hypoperfusion. In a study by Gelfand et al., thirty seven percent of patients had perfusion $C T$ abnormalities in post-ictal state, with prolonged MTT and decreased cerebral blood volume (CBV) and cerebral blood flow (CBF) being the most common.8 The cerebral blood flow changes during the course of seizure, from marked increase in ictal to a deep decrease in postictal state. ${ }^{9}$ Due to these findings, we conclude that our patient with prolonged MTT was in the postictal state during his CT on admission. Nonketotic hyperglycemia related occipital lobe seizures have been associated with subcortical hypointensity in occipital lobes on T2 weighted MRI. This is different from the hyperintensity of the occipital region often found in PRES.7 When comparing several case reports of nonketotic hyperglycemia induced occipital seizures, ten out of eleven patients had this characteristic transient hypointensity of the occipital region. 4 As far as we know, this is only the second case reporting nonketotic hyperglycemia induced occipital lobe seizures without MRI changes.

The pathology behind nonketotic hyperglycemia induced occipital lobe seizures is still unclear. It has been hypothesized that the seizure threshold decreases due to metabolic disturbance induced by chronic hyperglycemia. ${ }^{6}$ Another hypothesis predicts that previously existing cortical lesions lead to seizure under the metabolic disturbance, ${ }^{6}$ and pre-existing lesions may predispose a certain population to focal seizures with hyperglycemia, ${ }^{10}$ such as with our patient who showed chronic small vessel ischemic change on MRI. Also, the neuronal potassium ATP channels may be related to the neuroexcitability in the hyperglycemic state. ${ }^{10}$

In conclusion, our patient continued to seize for three days despite increasing dosage of AED. While in the hospital, he was also treated for his hyperglycemia, which we believe ultimately resulted in the gradual resolution of his seizures. The typical time elapsed from glycemic control to seizure cessation ranges from 24 hours to four days. 5 He has 
continued the AED regimen as well as antidiabetic therapy for 15 months post admission, keeping $\mathrm{HbA} 1 \mathrm{c}$ level under $7.8 \%$ with no seizure recurrence. Per Scherer, AED therapy should not be initiated or continued once seizures are under control.5 However, one case reported recurrence of occipital lobe seizures nine months after primary admission with loss of diabetic control without AED therapy. 3 We propose that prophylactic therapy may be necessary until $\mathrm{HbA} 1 \mathrm{c}$ is in the acceptable range. Our patient has attained glucose control, and a trial period without AED should be initiated.

\section{AUTHOR AFFILIATIONS}

1. Marshall University Joan C. Edwards School of Medicine, Huntington, West Virginia

\section{REFERENCES}

1. Adcock J, Panayiotipoulos P. Occpital lobe seizures and epilesies. Journal of Clinical Neurophysiology. 2012;29(5):11.

2. Taylor I. Occipital epilepsies: identification of specific and newly recognized syndromes. Brain. 2003;126(4):753-769.

3. Wang CP et al. Hyperglycemia with occipital seizures: images and visual provoked potentials. Epilepsia. 2005;46(7):5.

4. Hung WL et al. Occipital lobe seizures related to marked elevation of hemoglobin A1C: report of two cases. Seizure. 2010;19(6):359-362.

5. Scherer C. Seizures and non-ketotic hyperglycemia. Presse Med. 2005;34(15):6.

6. Wang $X$ et al. Nonketotic hyperglycemia-related epileptic seizures. Epilepsy Behav Case Rep 1. 2013:77-78.

7. Sasaki F et al. Occipital lobe seizures and subcortical T2 and T2 hypointensity associated with nonketotic hyperglycemia: a case report. J Med Case Rep. 2016;10(1):228.

8. Gelfand JM et al. Cerebral perfusion-CT patterns following seizure. Eur J Neurol. 2010;17(4):594601.

9. Wiest $\mathrm{R}$ et al. Detection of regional blood perfusion changes in epileptic seizures with dynamic brain perfusion CT--a pilot study. Epilepsy Res. 2006;(2-3):102-110.
10. Moien-Afshari F, Tellez-Zenteno JF. Occipital seizures induced by hyperglycemia: a case report and review of literature. Seizure. 2009;18(5):382385. 